\title{
Registro de ensayos con vacunas del Programa Mundial de Vacunas e Inmunización de la OMS ${ }^{1}$
}

\author{
S. E. Robertson, ${ }^{2}$ M. Vall Mayans, ${ }^{2,3}$ S. Horsfall, ${ }^{2}$ P. F. Wright, ${ }^{4}$ \\ J. Clemens, ${ }^{5}$ B. Ivanoff $^{2}$ y P. H. Lambert ${ }^{2}$
}

RESUMEN En 1995, el Programa Mundial de Vacunas e Inmunización de la OMS estableció un registro para ensayos con vacunas. En septiembre de 1996, este registro contenía 50 ensayos de vacunación patrocinados por la OMS, de los cuales 25 (50\%) eran estudios ya terminados. Las vacunas que se habian estudiado con mayor frecuencia fueron las de sarampión (9 ensayos), poliovirus ( 8 ensayos), cólera (8 ensayos), Escherichia coli enterotoxígena (4 ensayos) y neumococo (4 ensayos). Casi $80 \%$ de estos ensayos se llevaron a cabo en países en desarrollo, principalmente en el África. En los 25 ensayos ya terminados, los resultados investigados fueron la respuesta inmunitaria (24 ensayos), las reacciones adversas (13 ensayos), la morbilidad (4 ensayos) y la mortalidad (1 ensayo). La OMS contribuyó a estos ensayos con el aporte indirecto de fondos, ayuda con el diseño metodológico, visitas a las localidades, el análisis de los datos, la adquisición de vacunas y la investigación de su potencia.

El Programa Mundial de Vacunas e Inmunización (PMV) de la OMS se estableció en 1994. Con el fin de fortalecer su componente de investigación, se creó la Unidad del PMV para la

\footnotetext{
1 Se publica en inglés en el Bulletin of the World Health Organization, 1997, Vol. 75, No. 4, con el título "The WHO Global Programme for Vaccines and Immunization Vaccine Trial Registry". (c) Organización Mundial de la Salud, 1997.

2 Organización Mundial de la Salud, Programa Mundial de Vacunas e Inmunización, Ginebra, Suiza. Toda correspondencia y las solicitudes de separatas en inglés deben dirigirse a S. E. Robertson a la siguiente dirección postal: Organización Mundial de la Salud, 1211 Ginebra 27, Suiza.

3 Centro de Estudios Epidemiológicos sobre el SIDA en Cataluña, Badalona, Cataluña, España.

4 Universidad de Vanderbilt, Facultad de Medicina, Nashville, Tennessee, EUA.

5 Institutos Nacionales de Salud, Instituto Nacional de Salud Infantil y Desarrollo Humano, Bethesda, Maryland, EUA.
}

Investigación y el Desarrollo de Vacunas a base de combinar dos grupos de investigadores dedicados a estudiar estos productos: el Programa para el Desarrollo de Vacunas, perteneciente a la OMS y al Programa de las Naciones Unidas para el Desarrollo (PNUD) y responsable de un aporte anual de US\$ 3 a 5 millones para financiar la investigación de vacunas durante el período de 1984 a 1993 (1); y el componente de investigación del Programa Ampliado de Inmunización (PAI), que dio alrededor de US\$ 1 millón anuales para la investigación de vacunas durante el período de 1988 a 1993 (2).

Después del establecimiento de la Unidad de Investigación y Desarrollo de Vacunas del PMV, un panel de expertos externos recomendó crear un registro de los ensayos clínicos con vacunas a fin de mejorar el manejo interno de los estudios patrocinados por la OMS; calcular por anticipado los recursos necesarios para llevar a cabo estudios adicionales; identificar lugares en países en desarrollo que ya hubieran tenido experiencia en la conducción de ensayos clínicos con vacunas; y comparar las distintas metodologías que se aplican para realizar las investigaciones clínicas en torno a una vacuna. Hay buenos antecedentes con la creación de tales registros de ensayos, que permiten una apreciación general más eficiente de las investigaciones clínicas. La mayor parte de los registros se han creado con el propósito de facilitar las actividades de investigación, especialmente el fomento de estu- 
dios de mutua colaboración y multicéntricos, el uso de protocolos de investigación estandarizados y el mejoramiento de la metodología de estudio. Un repaso general reveló la presencia de 24 registros de ensayos que estaban activos en 1990 (3); cerca de la mitad de estos registros estaban destinados a estudios sobre los tratamientos contra el cáncer o el síndrome de inmunodeficiencia adquirida (sida), pero ninguno a los ensayos con vacunas.

A partir de 1990, el interés general por los ensayos clínicos con vacunas ha aumentado y se han creado varios registros destinados a ellos. En 1994, por ejemplo, la Comunidad Europea estableció un registro de ensayos clínicos con vacunas en el ámbito europeo (4); y la Colaboración Cochrane ha fomentado una serie de bases de datos y revisiones sobre tales ensayos $(5,6)$. En una revisión vinculada con la Colaboración Cochrane se examinaron el número y la calidad de los ensayos de vacunas publicados en la revista Vaccine (7).

Esta fuente actualizada describe el registro del PMV para ensayos con vacunas, que se estableció en 1995 (8). Se examinan el espectro de las vacunas y enfermedades abarcado por los ensayos incluidos en el registro; la distribución geográfica de los lugares donde estos se efectuaron; las características del diseño de los ensayos ya terminados; y las distintas contribuciones de la OMS a estos estudios.

\section{MATERIALES Y MÉTODOS}

El registro de ensayos de vacunas de la OMS/PNUD incluye ensayos de tres fuentes principales: los realizados o patrocinados por la OMS/PAI desde que se estableció en 1974 y hasta 1993; los realizados o patrocinados (o ambos) por el Programa de Desarrollo de Vacunas de la OMS de 1984 a 1993; y los realizados o patrocinados (o ambos) por la OMS/PMV a partir de 1994. La División de Salud y Desarrollo Humano también ha inscrito determinados ensayos con vacunas que a la larga serán fuentes de datos de interés para los administradores de progra- mas de inmunización. Hasta la fecha, el registro de la OMS/PMV no abarca los ensayos con vacunas patrocinados por el Programa Especial para la Investigación y Capacitación en Materia de Enfermedades Tropicales de la OMS, o por el Programa Conjunto de las Naciones Unidas sobre el VIH/ SIDA (ONUSIDA).

El registro solamente comprende estudios efectuados con seres humanos y abarca los siguientes: ensayos de vacunas que ya se han terminado; ensayos que aún están en proceso; ensayos que se espera iniciar en los próximos 12 meses; y ensayos que se han descontinuado. La información recogida para cada ensayo incluye los siguientes datos: el investigador principal; el país donde se llevó a cabo el ensayo; el tipo de vacuna que se estudió (incluidos el nombre del fabricante, el contenido de la vacuna y la vía de administración); la edad en el momento de la inscripción; el número de personas inscritas; el número de personas que llegaron a completar el estudio; el tipo de estudio; el método usado para asignar a los sujetos; el tipo de grupo usado para fines de comparación; el método de enmascaramiento; la duración del seguimiento; las variables de resultado examinadas; las instituciones que colaboraron en el estudio; las contribuciones de la OMS; y una lista detallada de las publicaciones vinculadas con el estudio.

Los datos del registro se resumieron a partir de fuentes de la OMS, tales como propuestas de estudio, informes anuales e informes sobre visitas a las localidades. También se entrevistó al funcionario de la OMS a cargo de dirigir cada estudio. Cuando quedaron dudas, estas se resolvieron por consulta con el investigador principal. Los datos correspondientes a cada ensayo se anotaron en un formulario uniforme y se creó una base de datos. Los datos del registro se analizaron con el programa Epi Info (9).

Para cada estudio se tomó como fecha de inicio el día en que se inscribió al primer paciente. Se clasificaron los países según el esquema de la OMS, que desglosa las economías en: industrializadas, en transición (aquí se incluye casi todo el territorio de la antigua Unión Soviética), o en desarrollo (10). Usamos los nombres de los fabricantes de las vacunas, tal como se notificaron a la OMS (11): en algunos casos, estos nombres indican que posteriormente se produjo una fusión entre una compañía farmacéutica cuya vacuna se estaba estudiando y otra compañía.

La clasificación de los ensayos con vacunas se basó en un esquema uniforme propuesto por Begg y Miller, con algunas modificaciones (12). Las evaluaciones de vacunas efectuadas antes de la autorización oficial se clasificaron en fase I (seguridad en voluntarios adultos), fase II-A (inmunogenicidad y reactogenicidad en la población destinataria), fase II-B (eficacia determinada por administración a voluntarios) y fase III (eficacia protectora). Las evaluaciones de las vacunas después de su autorización oficial se clasificaron en fase IV. El enmascaramiento fue a doble ciego (tanto el sujeto estudiado como el investigador desconocían qué vacuna se había administrado), a simple ciego (el sujeto estudiado era el desconocedor), o abierto (sin cegamiento) (13). Las variables de resultado se clasificaron en respuesta inmunitaria, reacciones adversas, morbilidad y mortalidad.

\section{RESULTADOS}

Para septiembre de 1996, 50 ensayos se habían incluido en el registro de ensayos con vacunas del PMV de la OMS. A partir de esa fecha, 25 (50\%) de los ensayos de vacunas ya habían terminado, 16 (32\%) estaban en marcha y 7 (14\%) estaban programados para comenzar en los próximos 12 meses (cuadro 1). Dos ensayos (4\%) - uno de fase III para la vacuna contra el cólera y otro de fase I para la vacuna contra el poliovirus - se descontinuaron por razones de metodología, pero aquí se examinan los 50 ensayos en su totalidad.

Un ensayo comenzó en 1982. En total se iniciaron 21 ensayos entre 1987 y 1993, equivalente a un promedio de tres ensayos nuevos cada año (figura 
CUADRO 1. Estado de los ensayos clínicos incluidos en el registro de ensayos con vacunas del Programa Mundial de Vacunas e Inmunización de la OMS, hasta septiembre de 1996

\begin{tabular}{|c|c|c|c|c|c|}
\hline $\begin{array}{l}\text { Vacuna } \\
\text { contra: }\end{array}$ & $\begin{array}{l}\text { No. de ensayos } \\
\text { terminados }\end{array}$ & $\begin{array}{l}\text { No. de ensayos } \\
\text { en marcha }\end{array}$ & $\begin{array}{l}\text { No. de ensayos } \\
\text { programados }\end{array}$ & $\begin{array}{l}\text { No. de ensayos } \\
\text { descontinuados }\end{array}$ & Total \\
\hline Cólera & 2 & 3 & 2 & 1 & 8 \\
\hline Dengue & - & 1 & - & - & 1 \\
\hline ECET $^{a}$ & 4 & - & - & - & 4 \\
\hline Hepatitis B & 1 & - & - & - & 1 \\
\hline $\mathrm{Hib}^{\mathrm{b}}$ & 1 & 1 & 1 & - & 3 \\
\hline Sarampión & 7 & 2 & - & - & 9 \\
\hline Meningococo & - & 3 & - & - & 4 \\
\hline Neumococo & 1 & 2 & 2 & - & 4 \\
\hline Poliovirus ${ }^{c}$ & 7 & - & - & 1 & 8 \\
\hline Rotavirus & 1 & 1 & - & - & 2 \\
\hline Tétanos & - & 1 & 1 & - & 2 \\
\hline Tuberculosis & 1 & - & - & - & 1 \\
\hline Fiebre tifoidea & - & 2 & 1 & - & 3 \\
\hline Total & 25 & 16 & 7 & 2 & 50 \\
\hline
\end{tabular}

${ }^{a}$ Escherichia coli enterotoxígena.

${ }^{\mathrm{b}}$ Haemophilus influenzae tipo B.

c Incluye un ensayo que se inició en 1982

1). Solamente un ensayo se inició en 1994, año en que se montó el PMV. En 1995 se iniciaron 11 ensayos nuevos en total; en 1996, 13; y en 1997, 3.

\section{Vacunas estudiadas}

En términos generales, las vacunas estudiadas con mayor frecuencia fueron las vacunas contra el sarampión (nueve ensayos), el poliovirus (ocho ensayos) y el cólera (ocho ensayos)

FIGURA 1. Número de ensayos incluidos en el registro de ensayos con vacunas del Programa Mundial de Vacunas e Inmunización de la OMS, según el año en que se iniciaron o se pensaba iniciarlos, sobre la base de informes recibidos hasta septiembre de 1996

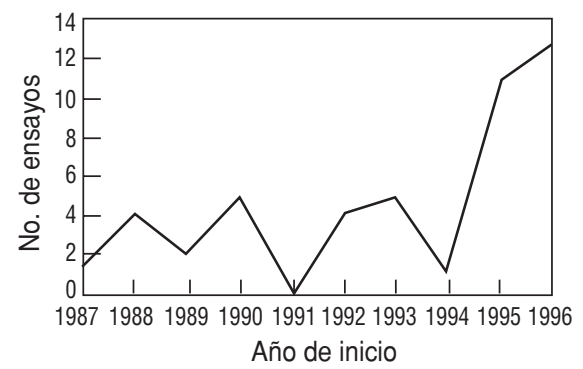

(cuadro 1). Las enfermedades que fueron objeto de estudio antes y después de 1994 fueron distintas (figura 2). Antes de 1994 la mayor parte de los ensayos tuvieron por objeto mejorar las vacunas normales de la infancia, y las que se investigaron con mayor frecuencia fueron las del sarampión (ocho ensayos) y la poliomielitis (siete ensayos). A partir de 1994, se ha prestado mayor atención a la evaluación de vacunas nuevas, de las cuales las más investigadas fueron las vacunas para el cólera (seis ensayos), infecciones neumocócicas (cuatro ensayos), Escherichia coli enterotoxígena (ECET) (cuatro ensayos), infecciones meningocócicas (tres ensayos) y fiebre tifoidea (tres ensayos) (figura 2).

FIGURA 2. Número de ensayos, por tema y período de inicio, incluidos en el registro de ensayos con vacunas del Programa Mundial de Vacunas e Inmunización de la OMS, 1987-1993 y

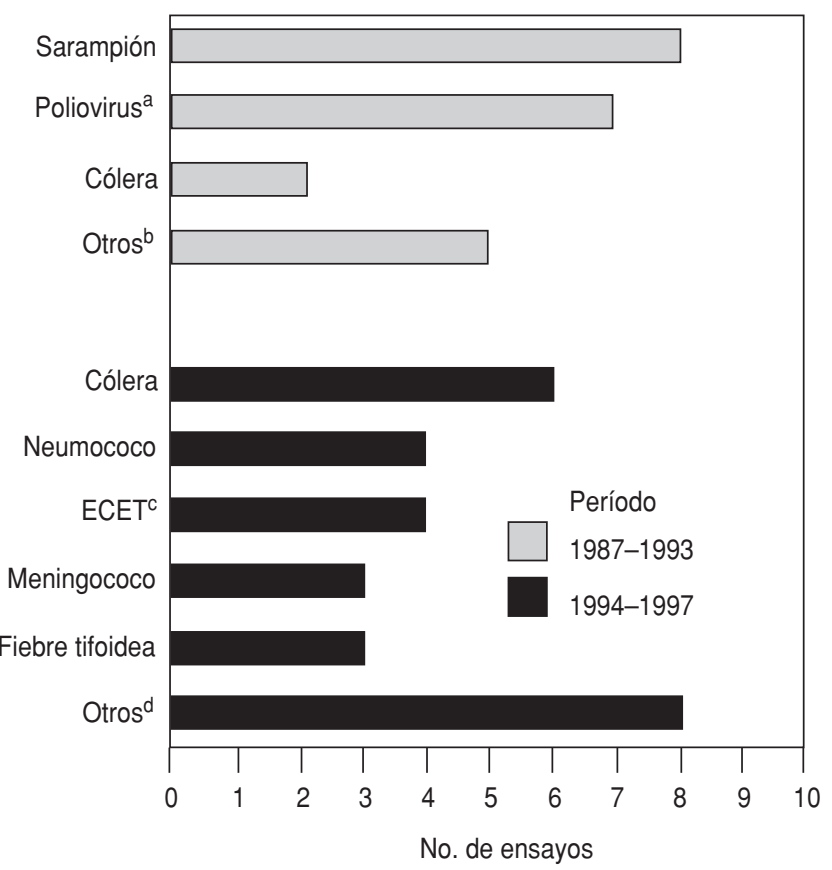

\footnotetext{
a Incluye un ensayo que empezó en 1982.

${ }^{\mathrm{b}}$ Hepatitis B (un ensayo); Haemophilus influenzae tipo b (1); meningococo (1); rotavirus (1); tuberculosis (1)

c Escherichia coli enterotoxígena.

d Haemophilus influenzae tipo b (dos ensayos); tétanos (2); dengue (1); sarampión (1); poliovirus (1); rotavirus (1)
} 
CUADRO 2. Características de las vacunas usadas o programadas para uso futuro en ensayos clínicos incluidos en el registro de ensayos con vacunas del Programa Mundial de Vacunas e Inmunización de la OMS, para septiembre de 1996

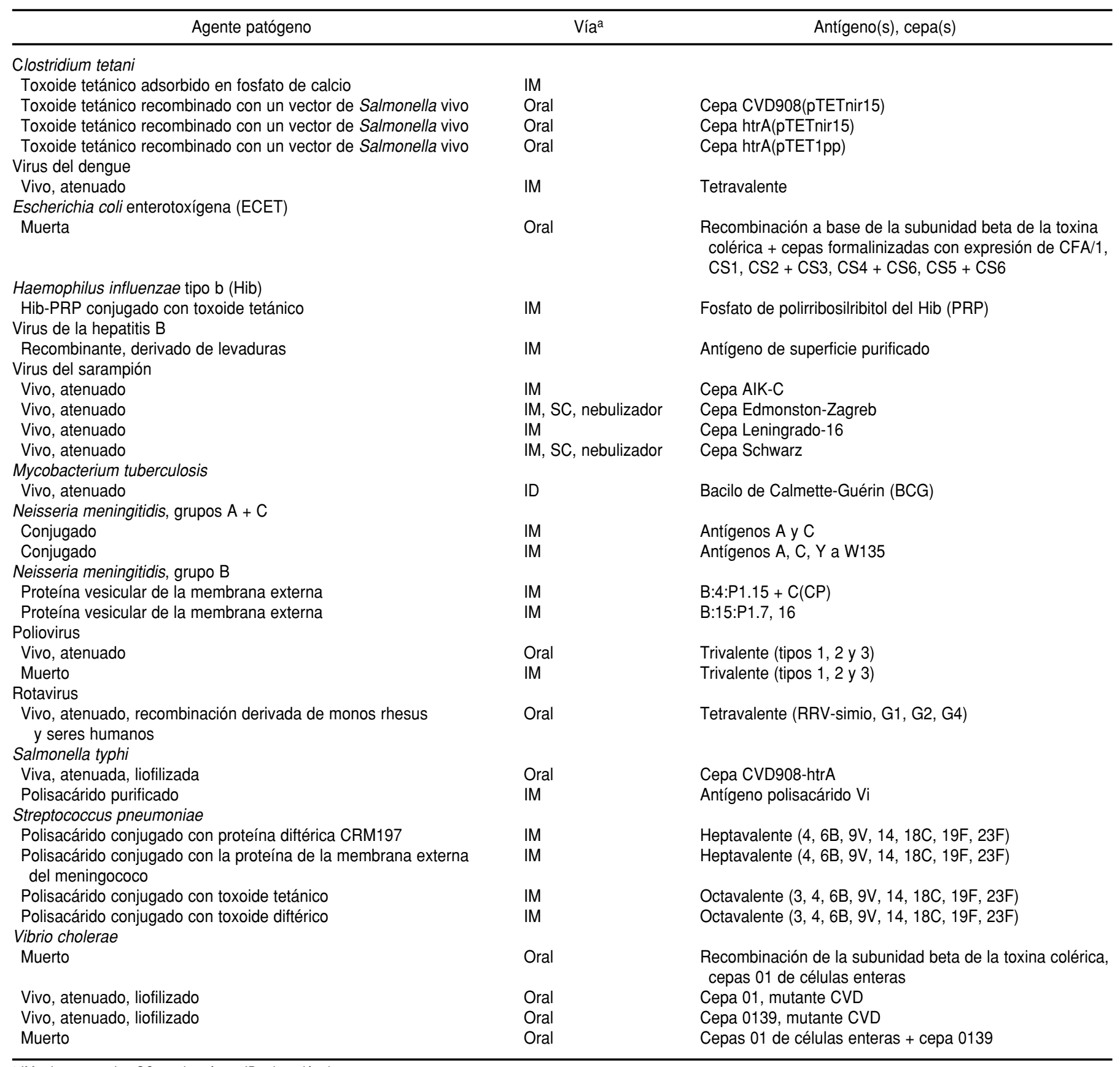

a $\mathrm{IM}$ = intramuscular; $\mathrm{SC}=$ subcutánea; $\mathrm{ID}$ = intradérmica.

En total, 30 vacunas candidatas se han sometido a ensayos incluidos en el registro (cuadro 2). De estas vacunas candidatas, 13 (43\%) fueron elaboradas para ser administradas por vías distintas de la habitual, ya fuese oralmente o por nebulizador.
Los 19 fabricantes de las vacunas candidatas sometidas a ensayos clínicos incluidos en el registro estaban ubicados en 15 países (cuadro 3), y cuatro $(27 \%)$ de ellos en países en desarrollo (Croacia, Cuba, Tailandia y Viet Nam).

\section{Lugares donde se efectuaron los ensayos}

De los 28 países que participaron en uno o más de los ensayos clínicos, 22 $(79 \%)$ eran países en desarrollo, uno (4\%) estaba en Europa oriental y cinco 
CUADRO 3. Fabricantes de vacunas candidatas investigadas o próximas a ser investigadas en ensayos clínicos incluidos en el registro de ensayos con vacunas del Programa Mundial de Vacunas e Inmunización de la OMS, según datos de septiembre de 1996

Biocine, Siena, Italia

Instituto Carlos J. Finlay, La Habana, Cuba

Center for Vaccine Development, Universidad de Maryland, Baltimore, MD, EUA

Centro para el Desarrollo de Vacunas, Universidad de Mahidol, Bangkok, Tailandia

Connaught Laboratories, Swiftwater, PA, EUA

Evans Medical, Liverpool, Reino Unido

Instituto de Inmunología, Zagreb, Croacia

Instituto de Poliomielitis y Encefalitis Vírica, Moscú, Federación de Rusia

Instituto Nacional de Higiene y Epidemiología, Hanoi, Viet Nam

Instituto Kitasato, Tokio, Japón

Lederle-Praxis, Pearl River, NY, EUA

Medeva, Leatherhead, Reino Unido

Merck Research Laboratories, Whitehouse Station, NJ, EUA

Pasteur/Mérieux Serums et Vaccins, Lyon, Francia

Riijksinstituut voor Volksgezondheid en Milieuhygiene, Bilthoven, Países Bajos

SBL Vaccin AB, Estocolmo, Suecia

SmithKline Beecham, productos biológicos, Rixensart, Bélgica

Statens Institutt for Folkehelse, Oslo, Noruega

Instituto Suizo de Sueros y Vacunas, Berna, Suiza
Los pacientes se asignaron aleatoriamente en 15 ensayos y de forma secuencial en dos. En un ensayo la delegación se hizo por comunidades, pero los resultados se analizaron individualmente por sujeto.

Las personas que recibieron varias vacunas en distintas ramas de un mismo estudio fueron comparadas en 10 ensayos: seis fueron estudios abiertos y cuatro, estudios a doble o simple ciego. En siete ensayos las personas pertenecientes al grupo de comparación recibieron placebo. En tres ensayos se comparó la respuesta a la vacunación de grupos con distintos estados serológicos con respecto al virus de la inmunodeficiencia humana (VIH). En un ensayo se comparó al grupo de individuos que recibieron las mismas
(18\%) eran países industrializados (figura 3). Un total de 18 ensayos clínicos con vacunas se llevaron a cabo en la Región de África, 12 en la Región de las Américas, 8 en la Región del Mediterráneo Oriental, 6 en la Región de Asia Sudoriental, 4 en la Región de Europa y 2 en la Región del Pacífico Occidental. Los ensayos de vacunas se llevaron a cabo con mayor frecuencia en los Estados Unidos de América (6 ensayos), Gambia (4 ensayos) y Omán (4 ensayos).

Dos ensayos fueron estudios de colaboración mutua entre varios países $(14,15) ;$ todos los demás se llevaban o llevan a cabo en un solo país, pese a que varios se efectúan en más de una localidad dentro del mismo país.

\section{Aspectos del diseño de los ensayos concluidos}

En total, 25 ensayos se han llevado a cabo hasta el final (cuadro 4). De ellos, 12 fueron estudios efectuados antes de la autorización oficial (dos fueron estudio de fase I; cinco fueron estudios de fase II y cinco fueron estudios de fase III). Un total de 13 ensayos clínicos concluidos se llevaron a cabo con vacunas ya autorizadas.
FIGURA 3. Mapa con los países donde se efectuaron ensayos clínicos incluidos en el registro de ensayos con vacunas del Programa Mundial de Vacunas e Inmunización de la OMS, sobre la base de informes recibidos hasta septiembre de 1996

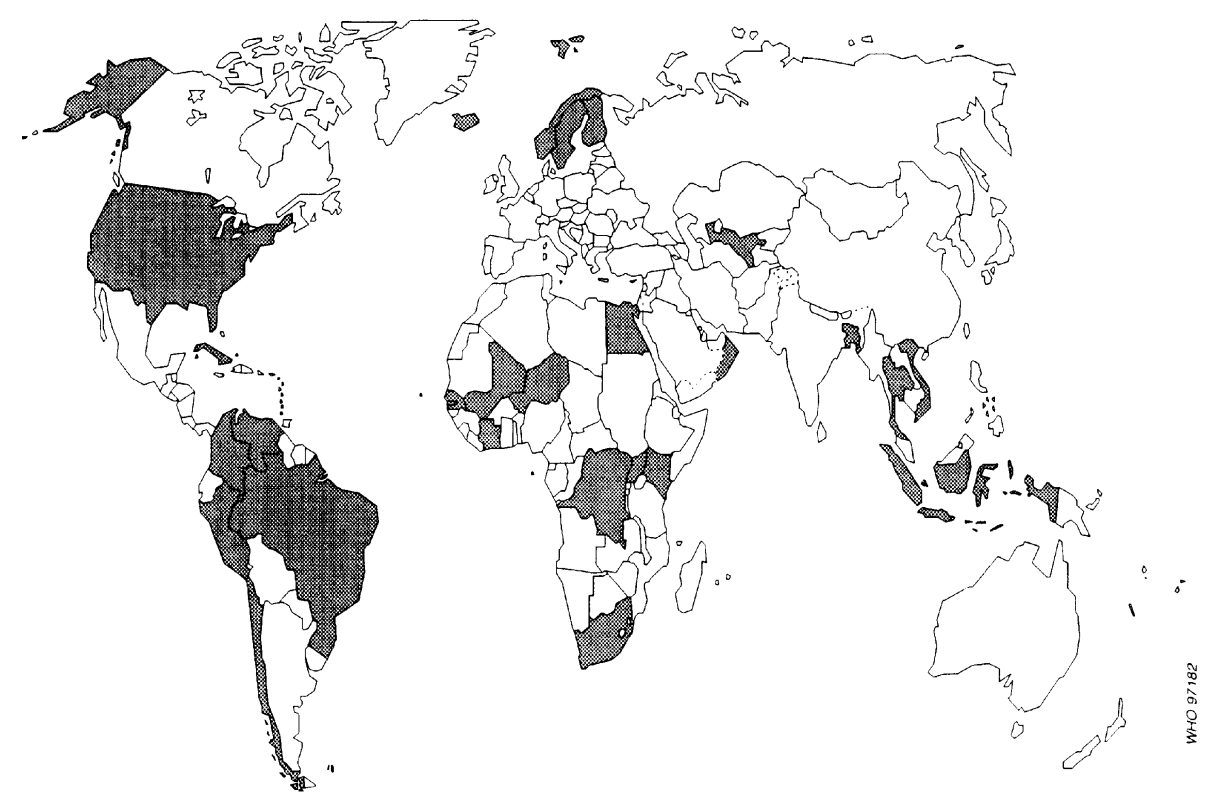

Nota: Los nombres y la presentación del material incluidos en este mapa no reflejan en absoluto ninguna opinión por parte de la Organización Mundial de la Salud con respecto a la existencia legal de ningún país, territorio, ciudad o zona, o de sus gobernantes, o a la delimitación de sus fronteras o límites territoriales. Las líneas quebradas son una representación aproximada de líneas fronterizas que en algunos casos podrían seguir siendo motivo de desacuerdo. 
CUADRO 4. Características de los estudios ya terminados que están incluidos en el registro de ensayos con vacunas del Programa Mundial de Vacunas e Inmunización de la OMS, según datos de septiembre de 1996

\begin{tabular}{|c|c|c|c|c|c|c|c|c|c|}
\hline $\begin{array}{l}\text { Vacuna } \\
\text { contra/país } \\
\text { del estudio }\end{array}$ & $\begin{array}{l}\text { Edad } \\
\text { al } \\
\text { entrar }\end{array}$ & $\begin{array}{l}\text { No. } \\
\text { inscrito }\end{array}$ & $\begin{array}{l}\text { Fase } \\
\text { del } \\
\text { estudio }\end{array}$ & $\begin{array}{l}\text { Designa- } \\
\text { ción de } \\
\text { sujetos }\end{array}$ & $\begin{array}{l}\text { Grupo de } \\
\text { compa- } \\
\text { ración }\end{array}$ & $\begin{array}{l}\text { Enmascara- } \\
\text { miento }\end{array}$ & $\begin{array}{l}\text { Duración del } \\
\text { seguimiento } \\
\text { hasta }\end{array}$ & $\begin{array}{l}\text { Resul- } \\
\text { tado }^{\mathrm{a}}\end{array}$ & Referencia \\
\hline Colombia & 1-64 años & 1933 & II-A & Aleatoria & Placebo & Doble ciego & $\begin{array}{l}2 \text { meses después } \\
\text { de la vacunación }\end{array}$ & $\mathrm{RI}, \mathrm{RA}$ & (30) \\
\hline Mali & Adulto & 84 & II-A & Aleatoria & Placebo & Doble ciego & $\begin{array}{l}3 \text { meses después } \\
\text { de la vacunación }\end{array}$ & $\mathrm{RI}, \mathrm{RA}$ & $\begin{array}{l}\text { C. Plowe, comunicación } \\
\text { personal, } 1996\end{array}$ \\
\hline \multicolumn{10}{|l|}{$\begin{array}{l}\text { Escherichia coli } \\
\text { enterotoxígena }\end{array}$} \\
\hline Bangladesh & Adulto & 27 & 1 & NA & NA & Abierto & $\begin{array}{l}5 \text { días después } \\
\text { de la vacunación }\end{array}$ & $\mathrm{RI}, \mathrm{RA}$ & $\begin{array}{l}\text { A-M Svennerholm, } \\
\text { comunicación personal, } \\
1996\end{array}$ \\
\hline Egipto & 6-12 años & 107 & II-A & Aleatoria & Placebo & Doble ciego & $\begin{array}{l}7 \text { días después } \\
\text { de la vacunación }\end{array}$ & $\mathrm{RI}, \mathrm{RA}$ & $\begin{array}{l}\text { S. Savarino, comunicación } \\
\text { personal, } 1996\end{array}$ \\
\hline \multicolumn{10}{|l|}{ Hepatitis B } \\
\hline Omán & 9 meses & 150 & IV & NA & NA & Abierto & 15 meses de edad & $\mathrm{RI}$ & $\begin{array}{l}\text { R. Sutter, comunicación } \\
\text { personal, } 1996\end{array}$ \\
\hline \multicolumn{10}{|l|}{$\begin{array}{l}\text { Haemophilus } \\
\text { influenzae tipo b }\end{array}$} \\
\hline Gambia & 8 semanas & 42848 & III & Aleatoria & Placebo & Doble ciego & 2,5 años de edad & $\begin{array}{l}\text { RI, RA } \\
\text { morbilidad }\end{array}$ & (32) \\
\hline \multicolumn{10}{|l|}{ Sarampión } \\
\hline Senegal & 5 meses & 2177 & IV & Aleatoria & Placebo & Simple ciego & $\begin{array}{l}8 \text { años después } \\
\text { de la vacunación }\end{array}$ & $\begin{array}{l}\text { RI, RA, } \\
\text { morbilidad, } \\
\text { mortalidad }\end{array}$ & (33) \\
\hline África del Sur & $6-9$ meses & 88 & IV & Secuencial & $\begin{array}{l}3 \text { grupos de } \\
\text { vacunas }\end{array}$ & Abierto & $\begin{array}{l}3 \text { meses después } \\
\text { de la vacunación }\end{array}$ & $\mathrm{RI}$ & (34) \\
\hline Uzbekistán & $6-9$ meses & 3677 & IV & Aleatoria & $\begin{array}{l}8 \text { grupos de } \\
\text { vacunas }\end{array}$ & Abierto & $\begin{array}{l}1 \text { año después } \\
\text { de la vacunación }\end{array}$ & $\mathrm{RI}, \mathrm{RA}$ & (35) \\
\hline Zaire & 6 meses & 301 & IV & NA & Histórico & Abierto & $\begin{array}{l}2 \text { años después } \\
\text { de la vacunación }\end{array}$ & $\begin{array}{l}\text { RI, cobertura } \\
\text { de la morbi- } \\
\text { lidad }\end{array}$ & (25) \\
\hline $\begin{array}{l}\text { Meningococo } \\
\text { Islandia }\end{array}$ & 15-20 años & 408 & II-A & Aleatoria & $\begin{array}{l}5 \text { grupos } \\
\text { de vacunas }\end{array}$ & Doble ciego & $\begin{array}{l}20 \text { meses después } \\
\text { de la vacunación }\end{array}$ & $\mathrm{RI}, \mathrm{RA}$ & (36) \\
\hline \multicolumn{10}{|l|}{ Poliovirus } \\
\hline$\left\{\begin{array}{l}\text { Brasil } \\
\text { Gambia }\end{array}\right.$ & $\{$ Al nacer & 2482 & III & $\left\{\begin{array}{l}\text { Secuencial } \\
\text { Aleatoria }\end{array}\right.$ & $\begin{array}{l}4 \text { grupos } \\
\text { de vacunas }\end{array}$ & Doble ciego & 6 meses de edad & $\mathrm{RI}$ & (14) \\
\hline Côte d'lvoire & $6-9$ meses & 808 & IV & Aleatoria & $\begin{array}{l}2 \text { grupos } \\
\text { de vacunas }\end{array}$ & Abierto & $\begin{array}{l}1 \text { año después } \\
\text { de la vacunación }\end{array}$ & $\mathrm{RI}$ & (29) \\
\hline Cuba & 0-3 años & 2087 & IV & NA & NA & Abierto & NA & $\mathrm{RI}$ & (37) \\
\hline
\end{tabular}


CUADRO 4. (Continuación)

\begin{tabular}{|c|c|c|c|c|c|c|c|c|c|}
\hline $\begin{array}{c}\text { Vacuna } \\
\text { contra/país } \\
\text { del estudio }\end{array}$ & $\begin{array}{l}\text { Edad } \\
\text { al } \\
\text { entrar }\end{array}$ & $\begin{array}{l}\text { No. } \\
\text { inscrito }\end{array}$ & $\begin{array}{c}\text { Fase } \\
\text { del } \\
\text { estudio }\end{array}$ & $\begin{array}{l}\text { Designa- } \\
\text { ción de } \\
\text { sujetos }\end{array}$ & $\begin{array}{l}\text { Grupo de } \\
\text { compa- } \\
\text { ración }\end{array}$ & $\begin{array}{l}\text { Enmascara- } \\
\text { miento }\end{array}$ & $\begin{array}{l}\text { Duración del } \\
\text { seguimiento } \\
\text { hasta }\end{array}$ & $\begin{array}{l}\text { Resul- } \\
\text { tado }^{a}\end{array}$ & Referencia \\
\hline$\left\{\begin{array}{l}\text { Gambia } \\
\text { Omán } \\
\text { Tailandia }\end{array}\right.$ & Al nacer & 1685 & IV & Aleatoria & $\begin{array}{l}3 \text { grupos } \\
\text { de vacunas }\end{array}$ & $\begin{array}{l}\text { Simple ciego } \\
\text { Doble ciego } \\
\text { Doble ciego }\end{array}$ & $\begin{array}{l}6 \text { meses de edad } \\
10 \text { meses de edad } \\
6 \text { meses de edad }\end{array}$ & $\mathrm{RI}$ & (15) \\
\hline Kenia & 2-3 meses & 200 & IV & Comunidad & $\begin{array}{l}3 \text { grupos } \\
\text { de vacunas }\end{array}$ & Abierto & 10 meses de edad & $\mathrm{RI}$ & (38) \\
\hline Omán & 9 meses & 1025 & IV & Aleatoria & $\begin{array}{l}4 \text { grupos } \\
\text { de vacunas }\end{array}$ & Abierto & 16 meses de edad & $\mathrm{RI}$ & $\begin{array}{l}\text { R. Sutter, comunicación } \\
\text { personal, } 1996\end{array}$ \\
\hline EUA & 2 meses & 108 & IV & Aleatoria & 2 itinerarios & Abierto & 7 meses de edad & $\mathrm{RI}$ & (39) \\
\hline \multicolumn{10}{|l|}{ Rotavirus } \\
\hline Venezuela & 2 meses & 2500 & III & Aleatoria & Placebo & Doble ciego & $\begin{array}{l}3 \text { años después } \\
\text { de la vacunación }\end{array}$ & Morbilidad & $\begin{array}{l}\text { N. Pierce, comunicación } \\
\text { personal, } 1996\end{array}$ \\
\hline \multicolumn{10}{|l|}{ Tuberculosis } \\
\hline Rwanda & Al nacer & 422 & IV & NA & 4 grupos & $\begin{array}{l}\text { Abierto } \\
\text { con distinta } \\
\text { serorreacti- } \\
\text { vidad a VIH }\end{array}$ & 15 meses de edad & $\mathrm{RI}, \mathrm{RA}$ & (26) \\
\hline
\end{tabular}

${ }^{\mathrm{a}} \mathrm{RA}=$ reacciones adversas; $\mathrm{RI}$ = respuesta inmunitaria; $\mathrm{NA}=$ No es aplicable.

vacunas como parte de esquemas diferentes, mientras que en otro se usaron controles históricos como grupo de comparación.

El número de sujetos en estudios varió de 27 a 42848 , con una mediana de 1 022. En 17 (68\%) de los 25 ensayos concluidos se inscribió a las personas menores de 12 meses de edad de la siguiente manera: neonatos (cuatro ensayos); niños de 2 a 3 meses (cuatro ensayos), niños de 5 a 9 meses (nueve ensayos). En tres ensayos se inscribió a niños; en cuatro, a adultos; y en uno, a personas de 1 a 64 años.

Como variables de resultado se midieron la respuesta inmunitaria (24 ensayos), las reacciones adversas (13 ensayos), la morbilidad (4 ensayos) y la mortalidad (1 ensayo). En la mayor parte de los ensayos se hizo un seguimiento de menos de 1 año a los sujetos. El único estudio en que se examinaron la morbilidad y mortalidad comprendió un seguimiento de 8 años; tres estudios en que solamente se examinó la morbilidad comprendieron períodos de seguimiento de 2 a 3 años.

Para septiembre de 1996, los 25 ensayos ya concluidos habían sido objeto de 56 publicaciones, y publica- ciones adicionales se estaban preparando en conexión con 10 de los ensayos, como mínimo. Todos los ensayos abarcaron varias instituciones colaboradoras (3,7 por ensayo, en promedio). Todos los ensayos en países en desarrollo se llevaron a cabo en colaboración con una o más instituciones ubicadas en países industrializados.

\section{La contribución de la OMS}

La OMS ha contribuido a los 50 estudios registrados mediante el financiamiento directo de 39 de ellos (78\%); asistencia con el diseño del estudio en 35 ensayos (70\%); el análisis de los datos en 22 ensayos (44\%); visitas a las localidades en 21 ensayos (42\%); la adquisición de vacunas en 17 ensayos (34\%), y la verificación de la potencia de la vacuna en 8 ensayos (16\%).

\section{DISCUSIÓN}

El registro de ensayos de vacunas del PMV de la OMS documenta un marcado aumento del número de ensayos efectuados desde la reorganización del programa. En 1987-1993, antes de dicha reorganización, se iniciaba anualmente un promedio de tres ensayos nuevos de vacunación; en 1995-1996, el promedio anual (12 ensayos al año) se había cuadruplicado. La capacidad ampliada del programa para administrar ensayos clínicos coincide con la aparición de un creciente número de vacunas candidatas. Se espera que aumente la demanda de ensayos para investigar vacunas, especialmente en países en desarrollo, y se proyecta que hasta 10 vacunas nuevas estarán ya listas para incorporarse en los esquemas habituales de vacunación en los próximos 10 años (16).

Casi $80 \%$ de los ensayos en el registro se efectuaron en países en desarrollo, y 18 en la Región de África de la OMS. Esto pugna con informes según los cuales los ensayos con vacunas se están realizando en países industrializados en conexión con enfermedades que afectan mayormente a los países en desarrollo (7). El registro abarca solamente los ensayos que cuentan con el apoyo de programas de la OMS, que han adquirido el compromiso de hacer frente a las necesidades en el nivel mundial mediante la prevención 
de las enfermedades transmisibles que acarrean las mayores cifras de morbilidad y mortalidad.

La mayor parte de las vacunas requieren pruebas sobre el terreno en varios contextos geográficos. En el caso de algunos microorganismos (entre ellos Haemophilus influenzae, los rotavirus, Salmonella typhi y Streptococcus pneumoniae), la cantidad de enfermedad que causan o el espectro de sus serotipos puede variar según la localidad (17-19). Aun en el caso de un agente infeccioso como el poliovirus, cuya naturaleza no parece cambiar, la respuesta a la vacuna puede variar en países en desarrollo y en países industrializados (20).

La mayor parte de los ensayos concluidos que figuran en el registro se llevaron a cabo con vacunas autorizadas que ya se estaban usando en programas nacionales de inmunización (la BCG, la vacuna contra la hepatitis B, la vacuna antisarampionosa y la vacuna contra el poliovirus). Estos ensayos se efectuaron con el fin de mejorar el contenido de las vacunas, simplificar su administración en términos operativos, o explorar la respuesta a la vacuna de grupos en alto riesgo, como el de las personas infectadas por VIH. Los ensayos efectuados después de la autorización han contribuido a refinar las recomendaciones de la OMS. Por ejemplo, cuando el PAI fue creado por la OMS a mediados de los años setenta, los esquemas de vacunación usados en países industrializados eran los recomendados para todos los países. Posteriormente, los resultados de estudios con vacunas ya autorizadas que se llevaron a cabo en países en desarrollo condujeron a modificaciones de los esquemas recomendados por la OMS para tales países, con una administración de las vacunas en edades más tempranas y con intervalos más breves entre las distintas dosis $(21,22)$.

Los estudios de eficacia con vacunas ya autorizadas, que pueden abarcar la evaluación del costo en función de su efectividad, tendrán importancia en el caso de vacunas nuevas que se anticipa serán incorporadas a los esquemas normales de vacunación $(23,24)$. Constituye un buen ejemplo el estudio patro- cinado por la OMS sobre la factibilidad de cambiar el esquema de inmunización antisarampionosa en Kinshasa, Zaire (25). En este estudio se examinaron diversos parámetros, entre ellos la respuesta serológica, factibilidad logística, aceptación por el público, cobertura alcanzada y cambios en la incidencia de casos de enfermedad notificados.

Los ensayos clínicos siguen siendo caros, no solo en términos de los fondos invertidos, sino también de la capacitación de personal, la administración en el terreno y el análisis de datos. Habitualmente se ha esperado que las instituciones que adquieren las habilidades necesarias para llevar a cabo ensayos clínicos sigan trabajando en este campo especializado de la investigación epidemiológica. El registro documenta seis instituciones que han efectuado varios ensayos de vacunas patrocinados por la OMS: Medical Research Council Laboratories, Fajara, Gambia; Centro para la Investigación sobre la Meningitis y la Esquistosomiasis, Niamey, Níger; Ministerio de Salud, Muscat, Omán; Centro para Ensayos con Vacunas, Universidad de Mahidol, Bangkok, Tailandia; Centro para el Desarrollo de Vacunas, Baltimore, MD, EUA; e Instituto Nacional de Salud y Epidemiología, Hanoi, Viet Nam. También se han documentado tres ejemplos en los que se efectuaron varios ensayos de vacunas con una misma cohorte. 1) En Rwanda, dos estudios sobre inmunogenicidad e inocuidad (con las vacunas BCG y antisarampionosa) se incluyeron en un estudio de cohortes de neonatos cuyas madres eran positivas a VIH $(26,27)$. 2) En Côte d'Ivoire se combinaron los ensayos aleatorios sobre diferentes vacunas antisarampionosas y distintas vacunas contra el poliovirus, aprovechando la importancia de administrar ambas vacunas durante una misma consulta $(28,29)$. 3) En Omán, los neonatos que participaron en un ensayo clínico aleatorio sobre distintas vacunas contra el poliovirus también se inscribieron en ensayos destinados a examinar su respuesta serológica a la vacuna contra la hepatitis B y contra el sarampión.

El registro solamente abarca estudios en que la OMS ha tenido participación directa y ha servido para funciones de administración interna. Según datos del registro, el programa reorganizado pronto dio su apoyo a un gran número de ensayos para investigar vacunas. Esto se debió en parte al establecimiento de un Comité Asesor del PMV sobre Epidemiología e Investigaciones sobre el Terreno y a la inclusión de epidemiólogos expertos en otros comités asesores de investigación que brindan su orientación al programa.

Varía el tipo de contribución que ha dado la OMS a los ensayos de vacunas. Aunque el apoyo financiero suele cubrir solamente una pequeña parte de los gastos asociados con un estudio, el respaldo dado por la OMS a un estudio determinado permite a los investigadores recaudar fondos de otras fuentes. Para poder aspirar a recibir fondos de la OMS, los investigadores deben ofrecer pruebas convincentes de que su trabajo ha sido aprobado en sus aspectos éticos por las autoridades nacionales y locales correspondientes, y su estudio debe tener la aprobación del Comité de la Secretaría de la OMS para las Investigaciones en Seres Humanos. La OMS ayuda a diseñar los estudios, a evaluar las localidades en que se realizan y a analizar los datos. Un aspecto especial en que vale la experiencia de la OMS es la negociación de acuerdos de colaboración entre países, entre ellos los que implican asociaciones entre países en desarrollo y países industrializados, así como ensayos de colaboración multinacionales. Se espera que la disponibilidad del Directorio del registro de ensayos con vacunas del PMV de la OMS (8) facilite futuras iniciativas de colaboración internacional para realizar ensayos con vacunas.

Agradecimiento. El registro de ensayos con vacunas del PMV de la OMS se creó con la orientación del Comité Asesor del PMS sobre Epidemiología e Investigaciones sobre el Terreno, encabezado por el profesor F. M. LaForce. Los fondos usados para establecer y mantener este registro fueron aportados por la Fundación Rockefeller, el gobierno de Suecia y el PNUD. 


\section{REFERENCIAS}

1. WHO/UNDP Programme for Vaccine Development. Report of the tenth session of the Scientific Advisory Group of Experts (SAGE). Geneva: World Health Organization; 1993. (Documento inédito MIM. PVD/ 93.8).

2. Robertson SE. Research and development within the Expanded Programme on Immunization: The first five years. Geneva: World Health Organization; 1993. (Documento inédito WHO/EPI/GEN/93.10).

3. Easterbrook PJ. Directory of registries of clinical trials. Stat Med 1992;11:345-423.

4. Moxon ER, et al. A common European network for clinical trials. En: Bjovatn B, ed. European Commission COST/STD Initiative on European Vaccine Research: Reports of the Expert Panels. Vaccine 1996;14:624-643

5. Fahey $\mathrm{T}$, et al. The type and quality of randomized controlled trials (RCTs) published in UK public health journals. J Public Health Med 1995;17:469-474.

6. Milne R, Thorogood M. Hand searching the Journal of Epidemiology and Community Health as part of the Cochrane Collaboration. J Epidemiol Community Health 1996; 50:178-181.

7. Jefferson $T$, Jefferson $V$. The quest for trials on the efficacy of human vaccines: Result of the handsearch of Vaccine. Vaccine 1996;14:461-464.

8. World Health Organization. WHO/GPV Vaccine Trial Registry Directory. Geneva: WHO; 1996. (Documento inédito WHO/ VRD/GEN/96.01).

9. Dean AG, et al. Epi Info, version 6: A word processing, database, and statistics program for epidemiology on microcomputers. Atlanta: Centers for Disease Control and Prevention; 1994.

10. United Nations. The sex and age distribution of the world populations: The 1994 revision. New York: UN; 1994.

11. World Health Organization. International list of availability of vaccines and sera. Geneva: WHO; 1996. (Documento inédito WHO/VSQ/95.02/suppl.1).

12. Begg N, Miller E. Role of epidemiology in vaccine policy. Vaccine 1990;8:180-189.

13. Hayes RB, et al. More informative abstracts revisited. Ann Int Med 1990;113: 69-76.

14. WHO Collaborative Study Group on Oral Poliovirus Vaccine. Factors affecting the immunogenicity of oral poliovirus vaccine: A prospective evaluation in Brazil and the Gambia. J Infect Dis 1995;171: 1097-1106.
15. WHO Collaborative Study Group on Oral and Inactivated Poliovirus Vaccines. Combined immunization of infants with oral and inactivated poliovirus vaccines: Results of a randomized trial in the Gambia. Oman, and Thailand. Bull World Health Organ 1996;74:253-268.

16. Lambert PH. Research priorities for vaccines to be used at a global level. Behring Inst Mitt 1994;95:1-6.

17. Wright PF. Approaches to prevent acute bacterial meningitis in developing countries. Bull World Health Organ 1989;67: 479-486.

18. Glass RI, Gentsch JR, Ivanoff B. New lessons for rotavirus vaccines. Science 1996;272:46-47.

19. Ivanoff B, Levine MM, Lambert PH. Vaccination against typhoid fever: Present status. Bull World Health Organ 1994;72: 957-971.

20. Patriarca PA, Wright PF, John TJ. Factors affecting the immunogenicity of oral poliovirus vaccine in developing countries: Review. Rev Infect Dis 1991;13: 926-939.

21. World Health Organization. Immunization policy. Geneva: World Health Organization; 1996. (Documento inédito WHO/ EPI/GEN/95.03 rev. 1).

22. Halsey N, Galazka A. The efficacy of DPT and oral poliomyelitis immunization schedules initiated from birth to 12 weeks of age. Bull World Health Organ 1985;63: 1151-1169.

23. Hall AJ, Aaby P. Tropical trials and tribulations. Int J Epidemiol 1990;19:777-781.

24. Clemens J, et al. Evaluating new vaccines for developing countries: Efficacy or effectiveness? JAMA 1996;275:390-397.

25. Cutts FT, et al. Measles control in Kinshasa, Zaire improved with high coverage and use of medium titre EdmonstonZagreb vaccine at age 6 months. Int J Epidemiol 1994;23:624-631.

26. BCG immunization and paediatric HIV infection. Wkly Epidemiol Rec 1992;67(18): 129-132.

27. Lepage $\mathrm{P}$, et al. Safety and immunogenicity of highdose Edmonston-Zagreb measles vaccine in children with HIV-1 infection: A cohort study in Kigali, Rwanda. Am J Dis Child 1992;146:550-555.

28. Moriniere B, et al. Serologic responses to two Edmonston-Zagreb measles vaccines in 6-month-old infants, and to Schwarz measles vaccine in 9-month-old infants. En: Program and Abstracts of the $32^{\text {nd }}$ Interscience Conference on Antimicrobial Agents and Chemotherapy, Anaheim, CA, USA 11-14 October 1992. Washington, DC: American Society for Microbiology; 1992. p. 180. (Resumen No. 416).

29. Moriniere BJ, et al. Immunogenicity of a supplemental dose of oral versus inactivated poliovirus vaccine. Lancet 1993;341: 1545-1550.

30. Concha A, et al. Safety and immunogenicity of oral killed whole cell recombinant B subunit cholera vaccine in Barranquilla, Colombia. Bull Pan Am Health Organ 1995; 29:312-321.

31. Ahren $\mathrm{C}$, et al. Intestinal antibody response after oral immunization with a prototype cholera B subunit-colonization factor antigen enterotoxigenic Escherichia coli vaccine. Vaccine 1993;11:929-934.

32. Mulholland K, et al. Randomised trial of Haemophilus influenzae type b-tetanus protein conjugate vaccine for prevention of pneumonia and meningitis in Gambian infants. Lancet 1997; 349:1191-1197.

33. Aaby $\mathrm{P}$, et al. Sex-specific differences in mortality after high-titre measles immunization in rural Senegal. Bull World Health Organ 1994;72:761-770.

34. Auwaerter PG, et al. Changes within T cell receptor V-beta subsets in infants following measles vaccination. Clin Immunol Immunopathol 1996;79:163-170.

35. Bolotovski VM, et al. Immunization of 6 and 9 month old infants with AIK-C, Edmonston-Zagreb, Leningrad-16 and Schwarz strains of measles vaccine. Int Epidemiol 1994;23:1069-1077.

36. Perkins BA, et al. Immunogenicity of two outer membrane protein-based serogroup B meningococcal vaccines among young adults in Iceland. En: Proceedings of the Ninth International Pathogenic Neisseria Conference, Winchester, England, 26-30 September 1994.

37. Mas Lago P, et al. Lessons from Cuba: Mass campaign administration of trivalent oral poliovirus vaccine and seroprevalence of poliovirus neutralizing antibodies. Bull World Health Organ 1994; 72:221-225.

38. Kok PW, et al. Serological and virological assessment of oral and inactivated poliovirus vaccines in a rural population in Kenya. Bull World Health Organ 1992, 70:93-103.

39. Cohen-Abbo A, et al. Seroresponse to trivalent oral poliovirus vaccine as a function of dosage interval. Pediatr Infect Dis J 1995;14:100-106. 
ABSTRACT In 1995, the WHO Global Programme for Vaccines and Immunization established a vaccine trial registry. As of September 1996, this registry included 50 WHOsupported vaccine trials, of which $25(50 \%)$ were completed studies. The vaccines most frequently tested have been against measles (9 trials), poliovirus ( 8 trials), cholera ( 8 trials), enterotoxigenic Escherichia coli (4 trials), and pneumococcus (4 trials). Nearly $80 \%$ of these trials have been conducted in developing countries, with the largest number being in Africa. Among the 25 completed trials, outcomes measured were immune response (24 trials), adverse reactions (13 trials), morbidity (4 trials), and mortality (1 trial). WHO's contributions to these studies include direct funding, assistance with study design, site visits, data analysis, vaccine procurement, and vaccine potency testing.

\section{The WHO Global Programme for Vaccines and Immunization Vaccine Trial Registry}

\section{Premio Fred L. Soper en Salud Interamericana, 1999}

\section{Fecha límite: 31 de marzo de 1999}

La Fundación Panamericana de la Salud y la Educación (PAHEF) solicita la recomendación de candidatos para este premio, con el cual se honra la memoria del doctor Fred L. Soper, ex Director de la OSP (1947-1958). Además de distinguirse por sus servicios sobresalientes en la OPS/OMS, en los años veinte y treinta el doctor Soper trabajó con la Fundación Rockefeller y desempeñó un papel protagónico en la lucha contra la fiebre amarilla y otras enfermedades infecciosas. Fue una de las figuras más destacadas del siglo en la salud interamericana.

El premio, que consiste en un diploma y US\$ 1000 , se concede anualmente a los autores de un trabajo científico publicado que aporte nuevos conocimientos al campo de la salud pública y sea de interés especial para América Latina, el Caribe, o ambos. El trabajo puede consistir en un informe basado en el análisis de nuevos datos de estudios experimentales o de observación, 0 en un enfoque novedoso de datos ya disponibles. Se dará preferencia a los estudios interdisciplinarios y a los que tratan de enfermedades infecciosas, tema de gran interés para el doctor Soper durante toda su vida. Han de considerarse solamente los trabajos publicados en revistas científicas incluidas en el Index Medicus. Además, el premio solo se concede a autores vinculados con instituciones docentes, de investigación o de servicio ubicadas en países de América Latina y el Caribe (incluidos los Centros de la OPS). Pueden concursar trabajos presentados por sus autores o en nombre de ellos, pero tienen que haberse publicado durante 1998. Todos los trabajos presentados deben recibirse a más tardar el 31 de marzo de 1999.

\section{Dirección para el envío de nominaciones: \\ Secretario Ejecutivo, PAHEF \\ 525 Twenty-third Street, NW \\ Washington, DC 20037, EUA}

